\title{
Economic analysis of waste recycle process in perhydropolysiloxazane synthesis
}

\author{
Huichan Yun, Seungjong Yeom, and Dae Ryook Yang ${ }^{\dagger}$ \\ Department of Chemical and Biological Engineering, Korea University, Anam-dong 5-ga, Seongbuk-gu, Seoul 136-713, Korea
} (Received 10 June $2013 \cdot$ accepted 8 December 2013)

\begin{abstract}
The perhydropolysiloxazane (PHPS) solution has been widely used in the spin-on-dielectric (SOD) process to form silicon oxide layer on a wafer in semiconductor industries. To reduce the whole semiconductor manufacturing cost, the process of PHPS solution production requires high productivity as well as low production cost. A large portion of the PHPS solution production cost is attributed to the large usage of solvents (pyridine and xylene), because more than 20 times of solvents in mass are required to produce a unit mass of high purity PHPS solution. Therefore, we suggest several plausible solvent regeneration processes of organic solvent waste from the PHPS solution production, and their economics is evaluated for comparison.
\end{abstract}

Keywords: Perhydropolysiloxazane, Recycle, Economic Analysis, Pyridine

\section{INTRODUCTION}

Shallow trench isolation (STI) layer is an element of a semiconductor and its role is to prevent electric signal interference, as a dielectric layer. In the past, chemical vapor deposition (CVD) or plasma enhanced chemical vapor deposition (PECVD) was employed to make the STI layer. However, these methods have two main demerits. First, they have low throughput although high investment and high operating cost are required. The other demerit is that void volume arises from overhang phenomenon when pitch size is less than $100 \mathrm{~nm}$. In the semiconductor industry, spin-on-coating method has been of interest for the past 20 years as an idea that could replace the CVD/PECVD processes. The basic concept of this technique is natural leveling when liquid is applied to surface topography. Particularly, spin-on-coating for STI layer production was called spin-onglass (SOG) at first, but now it is called, more generally, spin-ondielectric (SOD). The focus of this process is making a coating layer as close as possible to pure $\mathrm{SiO}_{2}$ by changing spin coating material or curing conditions [1]. Perhydropolysilazane is widely used as spin coating, but perhydropolysiloxazane (PHPS) is used instead in Samsung Cheil Industries.

The PHPS can be synthesized by reaction of ammonia and dichlorosilane with pyridine as solvent. However, this reaction produces ammonium chloride that causes some problems such as wafer defects when used in semiconductor manufacturing. Generally, the SOD coating solution should not contain more than $1 \mathrm{ppm}$ of ammonium chloride. Therefore, many purifying steps are required and large amount of solvents such as pyridine and xylene are needed to produce pure SOD coating solution. Actually, more than twenty times of solvent in mass is required to produce a unit mass of PHPS solution. Purchase expenses of pyridine and xylene account for large portion of manufacturing cost and also the disposal cost of organic waste solvent are about $\$ 400 /$ ton, which brings about additional

\footnotetext{
To whom correspondence should be addressed.

E-mail: dryang@prosys.korea.ac.kr

Copyright by The Korean Institute of Chemical Engineers.
}

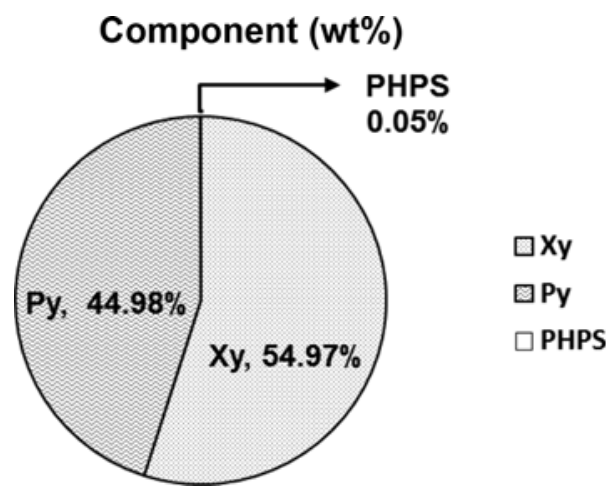

Fig. 1. The waste composition while producing SOD coating solution.

increase of SOD manufacturing cost.

Fig. 1 shows Pyridine/Xylene waste composition when pure SOD coating solution is produced. Almost all of waste is composed of pyridine and xylene. Trace (about $500 \mathrm{ppm}$ ) is the PHPS oligomer with uncontrolled molecular weight. Because PHPS is polymer in unstable state, even a small amount of PHPS in a waste tank can decompose to be silane, hydrogen, ammonia gases and that small amount of PHPS causes some safety problems. The occurrence of silane or other gases in the waste solution tank may lead to a very dangerous situation such as explosion if the gas concentration exceeds the limit of spontaneous combustion [2].

To prevent potential safety problems and to reduce the production cost, an efficient solvent regeneration process has to be developed. Thus, in this study, a regeneration process of organic solvent waste (pyridine and xylene) from the production process of SOD coating solution is suggested and economic analysis is performed.

\section{PROCESS}

\section{Decomposition of PHPS}

The solvent waste of SOD coating solution contains about 500 


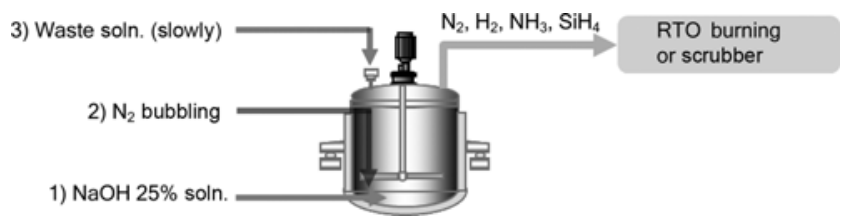

Fig. 2. Process schematic diagram of PHPS decomposition reactor.

ppm of PHPS. For the reuse of the solvent waste, the impure concentration of PHPS should be removed thoroughly. Otherwise, the remaining PHPS will cause defects for semiconductors and a hazardous situation in the storage tank of regenerated solvent due to the high reactivity of PHPS. Especially, among many kinds of gases produced by the decomposition of PHPS, silane has an autoignition limit of $14,000 \mathrm{ppm}$ in air. Thus, a process for the removal of PHPS has to be devised for the reasons mentioned above. However, the separation process by a conventional distillation will be very difficult and costly due to the broad polydispersity, as mentioned before. A new method is required. From the literature [3], it is reported that the PHPS can be decomposed by the following reaction:

$\mathrm{PHPS}+2 \mathrm{NaOH}+\mathrm{H}_{2} \mathrm{O} \rightarrow 2 \mathrm{H}_{2}+\mathrm{NH}_{3}+\mathrm{SiH}_{4}+\mathrm{Na}_{2} \cdot \mathrm{SiO}_{3}$

In Eq. (1), the Si-N bond of PHPS can react with sodium hydroxide and water to convert into hydrogen, ammonia, silane gas and sodium silicate in a controlled condition. Once it is decomposed, the gases can be removed easily by nitrogen bubbling. Also, the sodium silicate forms a water glass layer when dissolved in water, which can be removed easily by decantation or distillation. Thus, a PHPS decomposition process is proposed as in Fig. 2.

In this proposed process, $25 \%$ aqueous solution of sodium hydroxide is fed into the decomposition reactor. Then, nitrogen bubbling is initiated through a dip pipe for nitrogen purge to remove the dissolved gases efficiently. While nitrogen bubbling is maintained, the solvent waste containing PHPS is fed into the decomposition reactor slowly. At this point, the reactor temperature has to be maintained lower than the flash point of pyridine because the decomposition of PHPS and the mixing of water and waste are exothermic. The feeding rate of waste input should be controlled by monitoring the

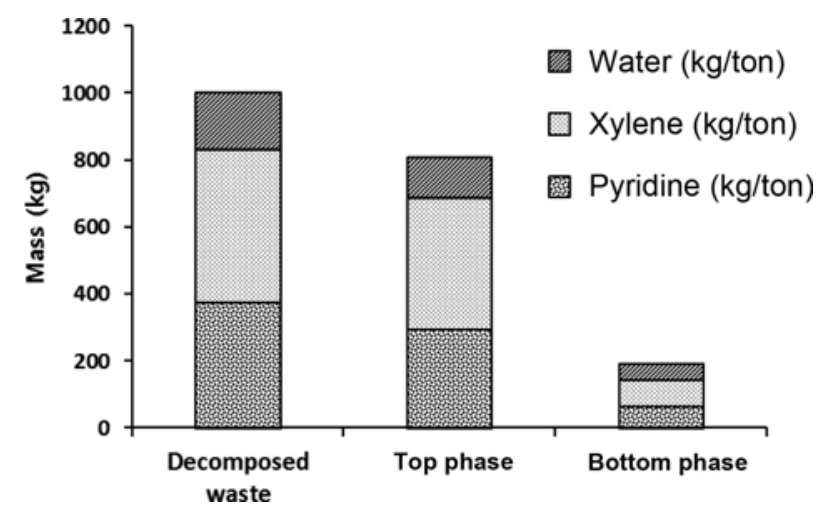

Fig. 4. Component proportion of decomposed waste and each separated phase.

amount of hydrogen and silane gases in the vent line by gas detector. The limit of hydrogen composition is $40,000 \mathrm{ppm}$ and that of silane is $14,000 \mathrm{ppm}$, and their limits should not be exceeded. This operation within the limits is very important for preventing the accidents. Especially, the level of silane needs to be carefully controlled because of its auto-ignition property.

\section{Purifying Process Design of PHPS-decomposed Waste Solu- tion}

The PHPS-decomposed waste solution can be purified by a separation system. Fig. 3 shows the schematic diagram of the purification process. The decomposition-completed waste is separated into two immiscible liquid phases in a decanter (one for water-rich phase and the other for xylene-rich phase). Fig. 4 shows the compositions of decomposed waste and each immiscible liquid phase. The amount of sodium silicate in the bottom phase is a trace, so that it was neglected in Fig. 4. Top phase and bottom phase are divided by about 8 -to- 2 in mass ratio. The bottom phase is a waste and the top phase will be sent to the solvent regeneration process. The amount of pyridine and xylene in the bottom phase will be sent to the solvent regeneration process. The high density bottom phase of decanter consists of water, pyridine, xylene and sodium silicate, and it can be removed easily by decantation. The removed bottom phase requires

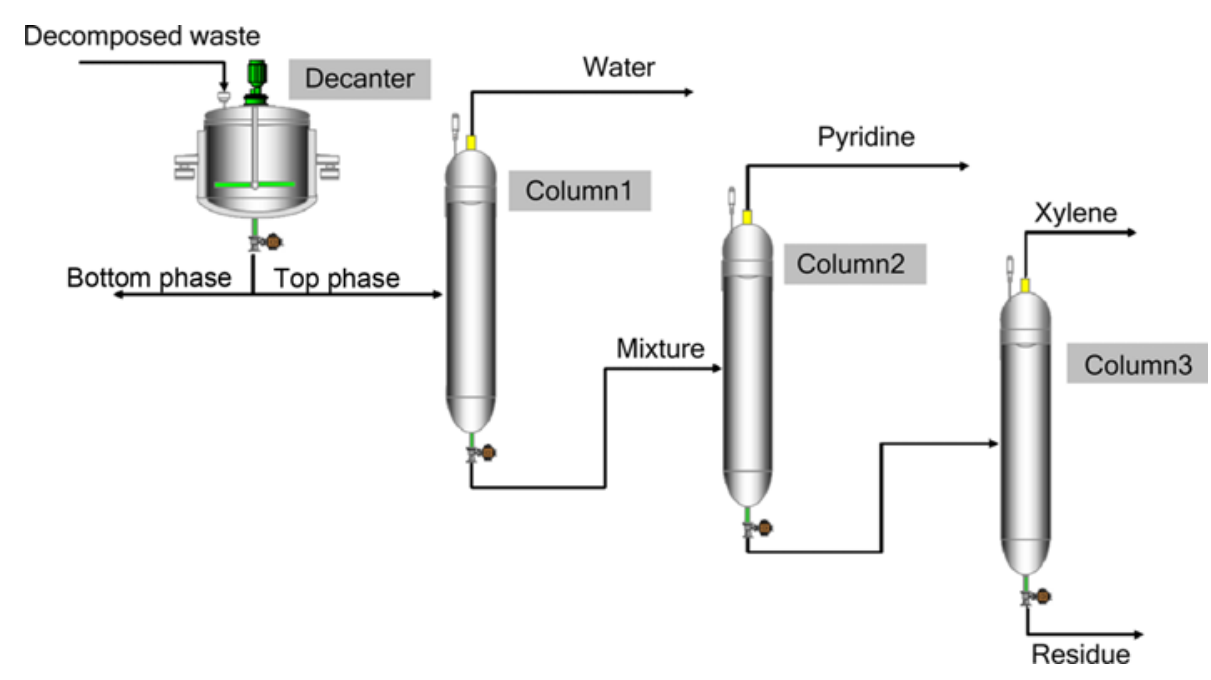

Fig. 3. Process schematic diagram of PHPS decomposed waste.

Korean J. Chem. Eng.(Vol. 31, No. 5) 
a neutralization treatment before it is sent to a waste water disposal facility.

The low density liquid phase in the upper part of decanter (mainly, water+pyridine+xylene) will be sent to the distillation sequence. In the first column, the water is separated as a distillate and the bottom product, which is a mixture of pyridine and xylene, will be sent to the second column. The first distillation tower can remove water over $99.5 \%$. In the second column, since the boiling point gap between pyridine and xylene is over $30^{\circ} \mathrm{C}$, the separation can be obtained easily. But the overhead pyridine stream contains a small amount of water, about $0.1 \%$. The water-contained pyridine may cause problems in using for SOD solution production directly. However, since the SOD production process has a unit process which can remove water by molecular sieve treatment with enough capacity, the water impurity can be removed without causing too much trouble.

\section{SIMULATION RESULT FOR PURIFICATION PROCESS}

Even though the specified purity of pyridine (xylene as impurity) and xylene (pyridine as impurity) for production of SOD in the semiconductor manufacture is about $99.99 \%$, a purity of more than $99.9999 \%$ is recommended in actual process considering the safety factor. In the purity specifications water is not considered as impurity because the SOD plant is equipped with molecular sieve module with enough capacity for water removal. For ultra-pure separation, a tall distillation tower will be required. However, the height of the distillation tower is limited to $20 \mathrm{~m}$ because Samsung Cheil Industries' SOD production system is an indoor plant. Due to this constraint, we chose the number of stages in the distillation column as forty in this study. While the total number of stages is fixed as forty, a series of simulations using Aspen Plus is conducted and checked whether pyridine and xylene of desired purity can be obtained. In this study, the simulations were performed using Aspen Plus in which NRTL and Redlich-Kwong (RK) models for liquid and gas phase are used, respectively. The NRTL model can estimate phase equilibrium of multi-component using binary interaction parameter only [4]. The objective of the first distillation column (column 1) is to limit the moisture content in the bottom product less than $1,000 \mathrm{ppm}$. If the moisture content exceeds $1,000 \mathrm{ppm}$, the molecular sieve column of SOD plant requires frequent regeneration for the moisture removal. The requirement for this specification is not severe and it is easily obtained without any difficulties. The second distillation column (column 2 ) is an atmospheric column, the feed rate is $500 \mathrm{~kg} / \mathrm{hr}$ at $25^{\circ} \mathrm{C}$, and the feed contains $49.44 \%$ of

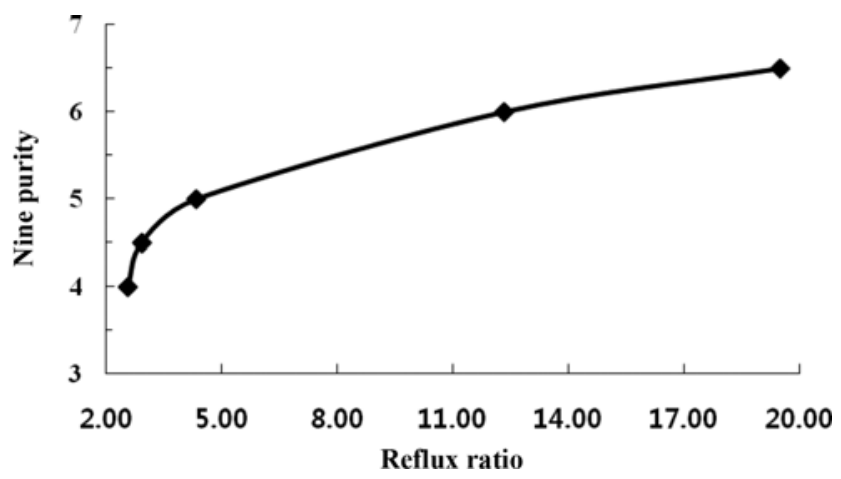

Fig. 5. Solvent purity as reflux ratio at 40-stage column 2 .

xylene with the rest being a mixture of pyridine and water. This feed rate is equivalent to 1,000 ton/year if the column is operated at 8 hours per day with 250 operating days per year. As mentioned above, the impurity specification for top product is six-nine purity. To satisfy the specification, the reflux ratio has been adjusted while the ratio between distillate and feed rates is fixed as 0.5045 , considering the feed concentration. Fig. 5 shows that the six-nine purity can be obtained by a forty-stage column when it is operated with 12.3 or higher reflux ratio. At this operating condition, the top and bottom temperatures are $114.2^{\circ} \mathrm{C}$ and $144.1^{\circ} \mathrm{C}$. Therefore, the regenerated solvent from the waste recycle process can be used in the PHPS synthesis process. The third column is for separating xylene from the residue which is mainly $\mathrm{NaOH}$. The separation can be achieved easily with a simple distillation column.

\section{ECONOMIC ANALYSIS}

For the economic analysis determining the feasibility of investment in the company, the fixed costs and variable costs should be considered. The fixed costs are the business expenses that are not dependent on the quality of products or services by the business. The variable costs are the expenses that change in proportion to the activity of a business [5]. The variable cost is the sum of marginal costs for total units produced. It can also be considered as normal cost. In this study, the fixed costs included investment and labor cost, and the variable costs included expenses for energy, QC, repair, maintenance, and consumables. The basic data for costing in this study was obtained from Samsung Cheil Industries. Also, the labor cost is considered as a fixed cost since a certain number of operating personnel are required regardless of the type of operation. The

Table 1. Basis of economic analysis for production rate of PHPS coating solution of 50 tons/year

(US Dollar)

\begin{tabular}{llllcr}
\hline \hline \multicolumn{2}{c}{ CAPEX } & & \multicolumn{2}{c}{ OPEX } & \\
\cline { 1 - 4 } Item & Cost & & Item & Double column case $^{a}$ & Triple column case $^{b}$ \\
\hline Decanter & $\$ 210,000$ & & Energy & $\$ 713$ & $\$ 826$ \\
Column 1 & $\$ 1,420,000$ & & QC & $\$ 161$ & $\$ 187$ \\
Column 2 & $\$ 1,270,000$ & & Consumables & $\$ 198$ & $\$ 244$ \\
Column 3 & $\$ 840,000$ & & Repair \& maintenance & $\$ 235$ & $\$ 375$ \\
Labor cost & $\$ 14,000 /$ Year & & Waste payment & $-\$ 730$ & $-\$ 1,351$ \\
\hline
\end{tabular}

${ }^{a}$ Double column case: regeneration cost per 1 ton of pyridine

${ }^{b}$ Triple column case: regeneration cost of pyridine 1 ton \& Xylene 1.3 ton

May, 2014 
parameters and basic data for the cost analysis are as listed in Table 1. Three cases are considered for comparisons. The first case is to regenerate both pyridine and xylene, which requires three columns for separation. The second case is to regenerate both pyridine and xylene, but they are used as cleaning solvent not for SOD production. The third case is to regenerate pyridine only, which requires two columns for separation. The equipment cost for the solvent regeneration plant was calculated by applying the six-tenths rule on the basis of current distillation column and the capacity of recycle process was calculated based on the maximum capacity of that plant. The six-tenths rule is given by

$$
\frac{\text { Cost }_{\text {plant } 2}}{\text { Cost }_{\text {plant } 1}}=\left(\frac{\text { Plant Capacity }_{\text {plant } 2}}{\text { Plant Capacity }}{ }_{\text {plant } 1}\right)^{m}
$$

where $\mathrm{m}$ denotes the size exponent. The correlation between normal capacity and cost used in engineering practice is the well-known power law rule. This law can be used to calculate the capital cost of a new plant (of known capacity) based on the known capital cost of an existing plant. The value for the exponent $\mathrm{m}$ is usually 0.6 , and thus it is called the "sixth tenths rule."

A convenient and plausible way for the comparison of investment decision is to use the capital recovery factor (CRF). The capital recovery factor gives the amount of money that must be repaid each year if the initial amount is borrowed. Of course, the factor depends on the duration of the loan and the interest rate [6]. A CRF is defined as a ratio of a constant annuity to the present value of receiving that annuity for a given length of time. Using an interest rate $i$, the capital recovery factor is defined as follow:

$$
\mathrm{CRF}=\frac{(\mathrm{i}+1)^{n}-1}{\mathrm{i}(\mathrm{i}+1)^{n}}
$$

where $\mathrm{n}$ is the number of annuities received.

For each case considered for the investment decision, the profit per year (PPY) can be calculated using Eq. (4) based on the process operating cost (POC) and labor cost (LC).

$$
\begin{aligned}
\mathrm{PPY} & =\text { batch No. } \times[(\mathrm{P} \times \text { unit price of } \mathrm{P}+\mathrm{X} \times \text { unit price of } \mathrm{X})-(\mathrm{POC}-\mathrm{W})] \\
& -\mathrm{LC}-\frac{\text { Investment cost }}{\mathrm{CRF}}
\end{aligned}
$$

where $\mathrm{P}$ and $\mathrm{X}$ are the amount of solvent regeneration per batch for pyridine and xylene, respectively, and $\mathrm{W}$ is the outsourcing cost for waste disposal when the regeneration process is not applied.

Since the devices for semiconductor processing are rapidly evolving, the semiconductor processing materials generally have short lifetime. Thus, for this feasibility investigation, the operating years in order to recover investment cost is estimated within ten years. The profit per years (PPY) of pyridine and xylene regeneration for the PHPS solution synthesis was calculated and plotted in Fig. 6 with respect to the plant life and annual batch number operated.

As expected, the profit will increase as the operation year is extended. However, due to the rapid changing characteristics of the semiconductor industry, it is reasonable to assume that the product lifetime is around five years if the manufacturing plant for PHPS is constructed with the regeneration process at the same time. If the waste regeneration process is added to an existing plant, the plant lifetime for the regeneration process will become even shorter. Thus,

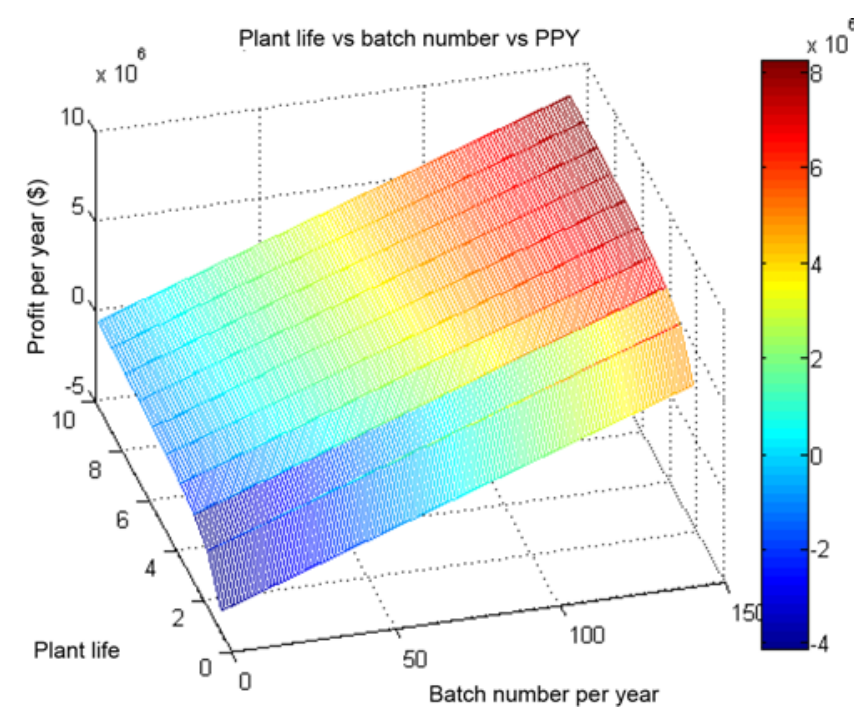

Fig. 6. Annual profit (PPY) with respect to batch number per year and plant life.

the lifetime for the waste regeneration plant is assumed to be four years.

\section{Case 1: Regeneration of Pyridine/Xylene for PHPS Solu-} tion Synthesis

The regenerated solvent from PHPS solution synthesis can be used in two ways: reuse for PHPS solution synthesis, and use for reactor cleaning. The case 1 is the former case. The reuse of regeneration solvents for PHPS solution synthesis has two merits. First, all the regeneration solvent can be reused for PHPS synthesis process and the new purchase of solvent will be reduced. If the regenerated solvent is used for cleaning, only a portion of regenerated solvent will be used for cleaning and rest of it will have to be sold or disposed. Second, since the decomposed waste has mainly two impurities (sodium hydroxide and sodium silicate), these impurities can almost be perfectly removed in the regeneration process and the regenerated solvents can have higher purity than the commercial solvent feeds which are synthesized pyridine and xylene stemmed from crude oil.

The lifetime of the regeneration process was fixed as four years, and the PPY (profit per year) was calculated according to batch num-

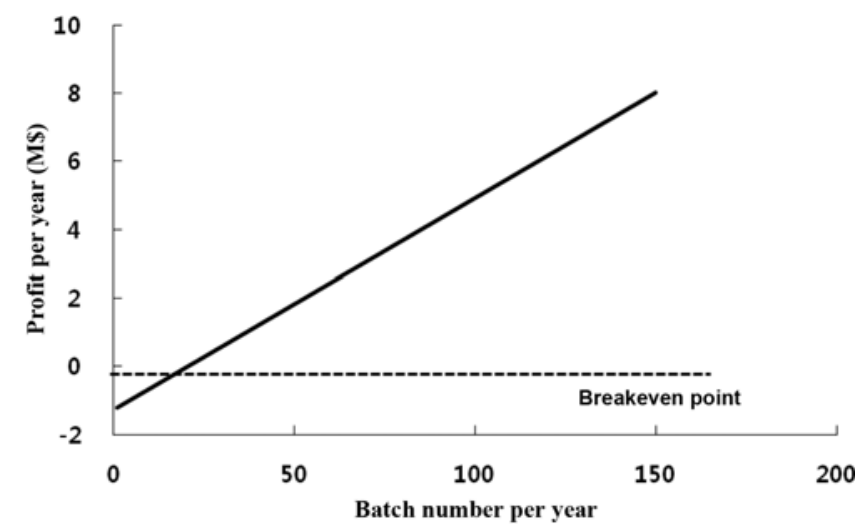

Fig. 7. Annual profit (PPY) with respect to batch number per year based on 4-year operation (case 1). 
bers for one year. Fig. 7 shows the result of calculation. According to Fig. 7, when 21 batches are operated per year, the breakeven point comes in four years. The reuse of recycled solvent for synthesis is economical because the current number of batches per year in PHPS manufacturing process of Samsung Cheil Industries is over 50 batches per year. The current production rate already exceeds the breakeven point of the waste recycle process.

\section{Case 2: Regeneration of Pyridine/Xylene for Reactor Clean- ing}

When an electronic device company purchases raw materials, the production history of the raw material is monitored in addition to quality inspection. Especially, semiconductor producers such as Samsung Electronics, SK Hynix, Toshiba monitor the quality of raw materials even from third-party vendors. The replacement of raw material is so significant that it cannot be executed without the agreement of the client. Even if raw material is changed with a client's consent, the product price may be discounted by the client's request. Case 1 may cause the problem explained above since the recycled solvent has different manufacturing history from that of the first vendor. Therefore, case 1 is hardly expected to be accepted by the client except for some serious situation such as lack of raw material supplier capacity.

Concerning this problem, the recycled solvent can be suggested to be used as cleaning solvent. In the general manufacturing process of PHPS for semiconductors, each batch should be cleaned by its own solvent. When water is used to clean the batch, it is very difficult to control moisture and reaction with the remaining PHPS. Currently, about eight tons of pyridine and 12 tons of xylene are required for producing one ton of PHPS solution. Additionally, two tons of pyridine and 1.5 tons of xylene are used for cleaning. Economic analysis is carried out when the amount of solvent for cleaning the reactor is supplied from the regenerated solvent and the rest of regenerated solvent is sold as bulk grade. In the same fashion as Case 1, profit per year (PPY) was calculated with respect to annual batch number and plotted in Fig. 8. As a result, when 49 batches are operated per year, the breakeven point comes in four years. Thus, the reuse of regenerated solvent for reactor cleaning will be profitable even if the current production rate is around the breakeven point since the expanding market will require more than hundred batches by sales forecast.

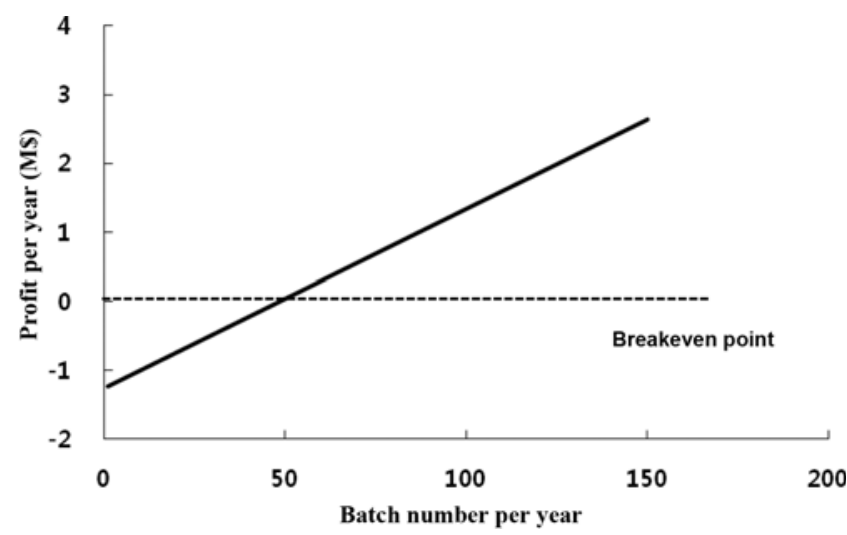

Fig. 8. Annual profit (PPY) with respect to batch number per year based on 4-year operation (case 2).

\section{Case 3: Regeneration of Pyridine Only}

Generally, there are two ways to produce pyridine commercially: to directly synthesize and purify pyridine, or to extract from coal and purify. The drawback of directly synthesized pyridine is that it contains impurities with similar boiling points, and thus they are not appropriate in semiconductor grade usage due to the difficulty of purification. On the other hand, the pyridine from coal can be purified through a comparably simple process to be semiconductorgrade pyridine of high purity. However, there is a limit on supply capacity because the pyridine from coal is a by-product from steel mills. For these reasons, the recycle of pyridine has more importance than xylene which is easily obtained from crude oil. If xylene is not reused in the regeneration process, the investment and energy costs can be saved while the cost for disposal of waste xylene is incurred. This case can be divided into two cases. One is to use regenerated pyridine for PHPS solution synthesis (case 3a), and the other for cleaning purpose (case 3b), while the xylene is not regenerated. The economic analysis for these cases was performed and illustrated in Fig. 9. According to Fig. 9, the operations of 21 and 57 batches per year will lead to the breakeven point for PHPS solution synthesis and for cleaning purposes, respectively. Considering that the market is expanding currently, these cases not only make profit but also provide alternatives in case of a price hike of pyridine.

In summary, the number of batches operated for four years and the accumulated profit for each case are depicted in Fig. 10. As men-

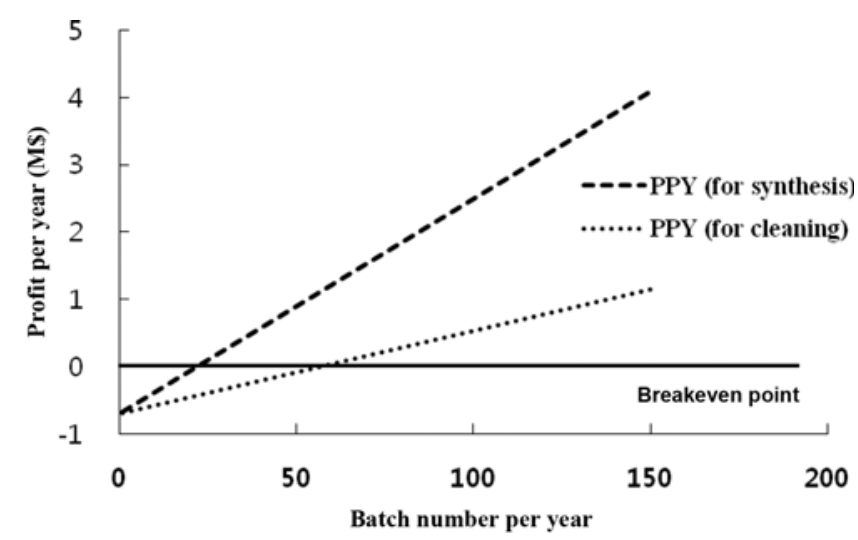

Fig. 9. Annual profit (PPY) with respect to batch number per year based on 4-year operation (case 3).

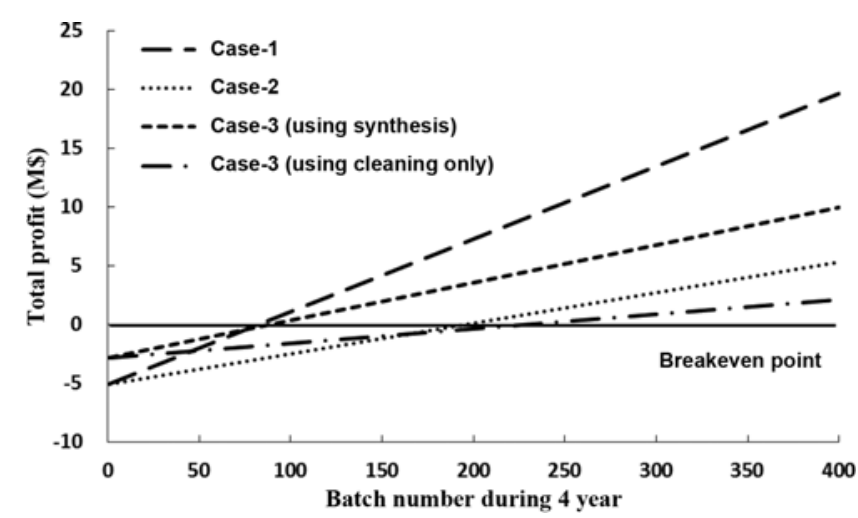

Fig. 10. Comparison of accumulate profit with respect to batch number per year based on 4-year operation. 
tioned earlier, cases 1, 2 and 3a will make a profit even with current production rate and case $3 \mathrm{~b}$ will exceed the breakeven point from the sales forecast. Therefore, whichever case is selected, the waste solvent regeneration process will be profitable in the production environment of the current PHPS manufacturing process. Among these alternatives, an appropriate case should be chosen after comprehensive consideration of investment cost, profit value, business environment and availability of each case.

\section{CONCLUSION}

To decide whether the waste solvent recycle system in the semiconductor PHPS manufacturing plant of Samsung Cheil Industries would be feasible or not, process simulations and economic analyses for different cases have been performed. The specification of the regenerated solvent to be reused requires 4-nine purity. However, the purity of 6-nine is industrially used for safety consideration of process operation. From the simulation, the 6-nine purity can be achieved under the given constraint which is the column height limitation.

Based on the economic analysis, the regeneration of both pyridine and xylene is most advantageous in the aspect of the breakeven point for profit. However, the possibility of solvent usage as cleaning purpose cannot be excluded due to the characteristics of electronic industries. Even in the least profitable case 3, where only pyridine is purified and it is used as cleaning solvent, 57 batches per year, i.e., 228 batches for four years can generate profit. Also, in this case, the initial investment cost is low and there can be potential cost saving at labor/supply expenses.

Even with the short lifetime of the waste regeneration process, the waste reuse will be profitable for all cases considered in this study. Therefore, waste regeneration in semiconductor PHPS production process is feasible. Furthermore, the waste regeneration process can be much more profitable when the amount of PHPS used in industry is expanded to other purposes, for example, car coating agent and oxygen barrier layer, and so on.

\section{ACKNOWLEDGEMENT}

This work was supported by the Human Resources Development program (No. 20134010200600) of the Korea Institute of Energy Technology Evaluation and Planning (KETEP) grant funded by the Korea government Ministry of Trade, Industry and Energy.

\section{REFERENCES}

1. J. A. Smythe, Spin-on-dielectrics: Planarity modeling, ProQuest, Ann Arbor (2009).

2. J. H. Park, T. S. Byun, S. Y. Jeon, S. Son, Y. H. Kwone, Y. Cho, J. D. Kim and M.-W. Kim, WO Application, 2011078446 (2011).

3. J. Gurman, J. E. Oberlander, T. Carl and B. Kicker, EP Patent, 2,024,432 (2009)

4. T. K. Kim, I. C. Jeon and S. T. Chung, Korean Chem. Eng. Res., 46(5), 903 (2008).

5. A. O'Sullivan and S. M. Sheffrin, Economics: Principles in Action, Pearson Prentice Hall, USA (2003).

6. B. Hazeltine and C. Bull, Field guide to appropriate technology, Academic Press, USA (2003).

7. J.-S. Bae, I. S. Hwang, Y.-J. Kweon, Y.-C. Choi, S. J. Park, H.-J. Kim, H. Jung and C. Han, Korean J. Chem. Eng., 29(7), 868 (2012).

8. Y. M. Park, H. W. Kang and Y. H. Kim, Theories Appl. Chem. Eng., 12(2), 1510 (2006).

9. R. Agrawal and Z. T. Fidkowski, AIChE J., 44(11), 2565 (1998).

10. M. B. Nikoo and N. Mahinpey, Biomass BioEnergy, 32, 1245 (2008).

11. M. Peters, K. Timmerhaus and R. West, Plant design and economics for chemical engineers, McGraw-Hill, USA (2002).

12. S.-J. Wang and D. S. H. Wong, Chem. Eng. Sci., 62, 1010 (2007).

13. J. S. Page, Conceptual cost estimating manual, Gulf Professional Publishing, USA (1996). 